\title{
APPENDIX 2: TABLES
}

Table 1: Tenants of Hugh de Lacy in England

From Cartae Baronum and Herefordshire Domesday, $1165^{-6^{1}}$

\begin{tabular}{|c|c|c|}
\hline \multicolumn{3}{|l|}{ Combined holdings } \\
\hline Tenant & Territory & $\begin{array}{l}\text { Service (new or old } \\
\text { feoffment) }\end{array}$ \\
\hline $\begin{array}{l}\text { Bacton (Bakington), } \\
\text { William of }\end{array}$ & $\begin{array}{l}\text { Bacton ( } 5 \text { hides) } \\
\text { Hampton [Court] ( } 2 \\
\text { hides) Ledicot ( } 1 \text { hide) } \\
\text { Letton ( } 3 \text { hides; HDB: } \\
\text { Adam) Wadetune (1 } \\
\text { hide; HDB: Gilbert) }\end{array}$ & 4 knights' fees (old) \\
\hline $\begin{array}{l}\text { Bartholomaeus } \\
\text { *Baskerville, Robert } \\
\quad \text { de }\end{array}$ & $\begin{array}{l}6 \text { librates of land } \\
\text { Brobury }^{2}(2 \text { hides) } \\
\text { Eardisley; Staunton- } \\
\text { on-Wye ( } 4 \text { hides) } \\
\text { Stretton Sugwas }(21 / 2 \\
\text { hides) Yarsop (1 } 1 \frac{2}{2} \\
\text { hides) Yazor ( } 5 \text { hides) }\end{array}$ & $\begin{array}{l}\text { No fixed service } \\
5 \text { knights' fees (old) }{ }^{3}\end{array}$ \\
\hline $\begin{array}{l}\text { Bret, Richard le } \\
\text { Briouze, W. de }\end{array}$ & King's Pyon (1/2 hide) & $\begin{array}{l}\text { No fixed service } \\
\text { (Not in cartae } \\
\text { baronum) }\end{array}$ \\
\hline Burford, Roland de & & 3 knights' fees (old) \\
\hline *Castello, Herbert de & & 2 knights' fees (new) \\
\hline $\begin{array}{l}\text { Caulton (Cauledone), } \\
\text { fee of }\end{array}$ & & $1 / 2$ knight's fee (new) \\
\hline $\begin{array}{l}\text { +Cormeilles, Richard } \\
\text { de }\end{array}$ & & 2 knights' fees (old) \\
\hline *Cuillardville, fee of & Wolferlow (61/2 hides) & 3 knights' fees (old) \\
\hline Criquetot fam. & Webton (21/2 hides) & $\begin{array}{l}\text { (Not in cartae } \\
\text { baronum) }\end{array}$ \\
\hline $\begin{array}{l}\text { Escorchebeuf, Roger } \\
\text { de }\end{array}$ & & No fixed service \\
\hline
\end{tabular}


Table 1: (continued)

\begin{tabular}{|c|c|c|}
\hline \multicolumn{3}{|l|}{ Combined holdings } \\
\hline Tenant & Territory & $\begin{array}{l}\text { Service (new or old } \\
\text { feoffment) }\end{array}$ \\
\hline Escotot, Anketil de & $\begin{array}{l}\text { Arkston ( } 1 \text { hide) } \\
\text { Cobhall ( } 1 \text { hide) }\end{array}$ & No fixed service \\
\hline *Escotot, Richard de & $\begin{array}{l}\text { King's Pyon ( } 5 \text { hides) } \\
\text { Wormsley (1 virgate) }\end{array}$ & 3 knight's fees (old) \\
\hline $\begin{array}{l}+ \text { Escotot, Roger de } \\
+ \text { Escotot, William de }\end{array}$ & & $\begin{array}{l}1 \text { knight's fee (new) } \\
\text { No fixed service }\end{array}$ \\
\hline $\begin{array}{l}*+\text { d'Évreux, fee of }^{\text {Walter }}\end{array}$ & $\begin{array}{l}\text { Elston (Elnodestone) (3 } \\
\text { hides) Lyonshall ( } 5 \\
\text { hides) }\end{array}$ & 3 knights' fees (old) \\
\hline +d'Évreux, Roger & & 4 knights' fees (old) \\
\hline +Ewyas, Roger of & & 1 knight's fee (old) \\
\hline Feipo, Adam de & & No fixed service \\
\hline $\begin{array}{l}\text { Fresne (Fraxino), } \\
\quad \text { Alvredus du }\end{array}$ & 1 virgate & No service listed \\
\hline +Fourches, William de & & $\begin{array}{l}2 \text { knights' fees (old) } \\
\text { (Denies another } 1 \\
\text { fee) }\end{array}$ \\
\hline $\begin{array}{l}\text { Gamaches, Godfrey } \\
\text { de }\end{array}$ & $\begin{array}{l}\text { Mansel Gamage ( } 8 \\
\text { hides) }\end{array}$ & 2 knights' fees (old) \\
\hline Gilh (?) & Marston (1/2 hide) & \\
\hline Hopton, William of & & 1 knight's fee (new) \\
\hline $\begin{array}{l}\text { Hungerford, Nicholas } \\
\text { of }\end{array}$ & & 4 knights' fees (old) \\
\hline Ley $($ Lega $)$, William of & $\begin{array}{l}\text { Almeley ( } 4 \text { hides }) \\
\text { Upcott ( } 1 \text { hide }) \\
\text { Woonton ( } 1 \text { hide })\end{array}$ & 3 knights' fees (old) \\
\hline $\begin{array}{l}\text { *Longchamp, Hugh } \\
\text { de }\end{array}$ & Marden (1 hide) & $\begin{array}{l}1 \text { knight's fee (old) } \\
\text { Fee of William de } \\
\text { Burhope, held in } \\
\text { maritagium }\end{array}$ \\
\hline $\begin{array}{l}\text { Macgne, son of } \\
\text { Gerard de }\end{array}$ & & No fixed service \\
\hline Malgeri (?) & $\begin{array}{l}\text { Grendon }(4 \text { hides }) \text { Or } \\
\text { Stanford }(1 \text { hide })^{4}\end{array}$ & \\
\hline Mans, Walter del & & 1 knight's fee (old) \\
\hline
\end{tabular}


Table 1: (continued)

\begin{tabular}{|c|c|c|}
\hline \multicolumn{3}{|l|}{ Combined holdings } \\
\hline Tenant & Territory & $\begin{array}{l}\text { Service (new or old } \\
\text { feoffment) }\end{array}$ \\
\hline Map, Walter & $\begin{array}{l}\text { Wormsley ( } 1 \text { hide, } \\
1 \text { virgate) }\end{array}$ & $\begin{array}{l}\text { (Not in cartae } \\
\text { baronum) }\end{array}$ \\
\hline Munsley, Rodland of & $\begin{array}{l}\text { Lyde [Mucegros?] } \\
\text { (1 hide) Munsley } \\
\text { ( } 3 \text { hides, } 1 \text { virgate) }\end{array}$ & 3 knights' fees (old) \\
\hline Odo (?) & Stoke Lacy (10 hides) & $\begin{array}{l}\text { (Not in cartae } \\
\text { baronum) }\end{array}$ \\
\hline Poer, R. le & $\begin{array}{l}\text { Lawton (in } \\
\text { Kingswood) (1 hide) }\end{array}$ & $\begin{array}{l}\text { (Not in cartae } \\
\text { baronum) }\end{array}$ \\
\hline Ralph (?) & Byford (5 hides) & $\begin{array}{l}\text { (Not in cartae } \\
\text { baronum) }\end{array}$ \\
\hline Richard fitz Roger & $\begin{array}{l}\text { Monkhide (Hid') } \\
\text { (1 hide) }\end{array}$ & $\begin{array}{l}\text { (Not in cartae } \\
\text { baronum) }\end{array}$ \\
\hline Riquetot, Ralph de & & No fixed service \\
\hline Roger fitz Odo & & 1 knight's fee (new) \\
\hline Sai, Elias de & & $\begin{array}{l}3 \text { knights' fees (old) } \\
\text { (Denies another } 1 \\
\text { fee) }\end{array}$ \\
\hline Sarnesfield, Philip de & $\begin{array}{l}\text { Sarnesfield (1 } 1 \frac{1}{2} \text { hides) } \\
\text { (HDB: Geoffrey) }\end{array}$ & $\begin{array}{l}1 / 2 \text { knight's fee }(\mathrm{old}) \\
\text { (Denies another } 1 / 2 \\
\text { fee) }\end{array}$ \\
\hline Saussey fam. & Lyde Saucey (2 hides) & $\begin{array}{l}\text { (Not in cartae } \\
\text { baronum - socage in } \\
1242-3)\end{array}$ \\
\hline Staunton, O. de & $\begin{array}{l}\text { Staunton-on-Wye } \\
\text { ( } 2 \text { hides })\end{array}$ & $\begin{array}{l}\text { (Not in cartae } \\
\text { baronum) }\end{array}$ \\
\hline Tyrell, son of Rocelin & $\begin{array}{l}\text { Evesbatch ( } 1 \text { hide) } \\
\text { (HDB: H. de Tyrell) } \\
\text { Little Marcle ( } 5 \text { hides) } \\
\text { (HDB O. de Tyrell) }\end{array}$ & 2 knights' fees (old) \\
\hline Underly, Adam of & $\begin{array}{l}\text { Underly (in Wolferlow) } \\
\text { (1/2 hide) }\end{array}$ & $\begin{array}{l}\text { (Not in cartae } \\
\text { baronum) }\end{array}$ \\
\hline William fitz Michael & & $\begin{array}{l}1 / 4 \text { knight's fee } \\
\text { (old) }\end{array}$ \\
\hline Wimund & $\begin{array}{l}\text { Street (Lestret in } \\
\text { Kingsland }\end{array}$ & $\begin{array}{l}\text { (Not in cartae } \\
\text { baronum) }\end{array}$ \\
\hline
\end{tabular}


Table 1: (continued)

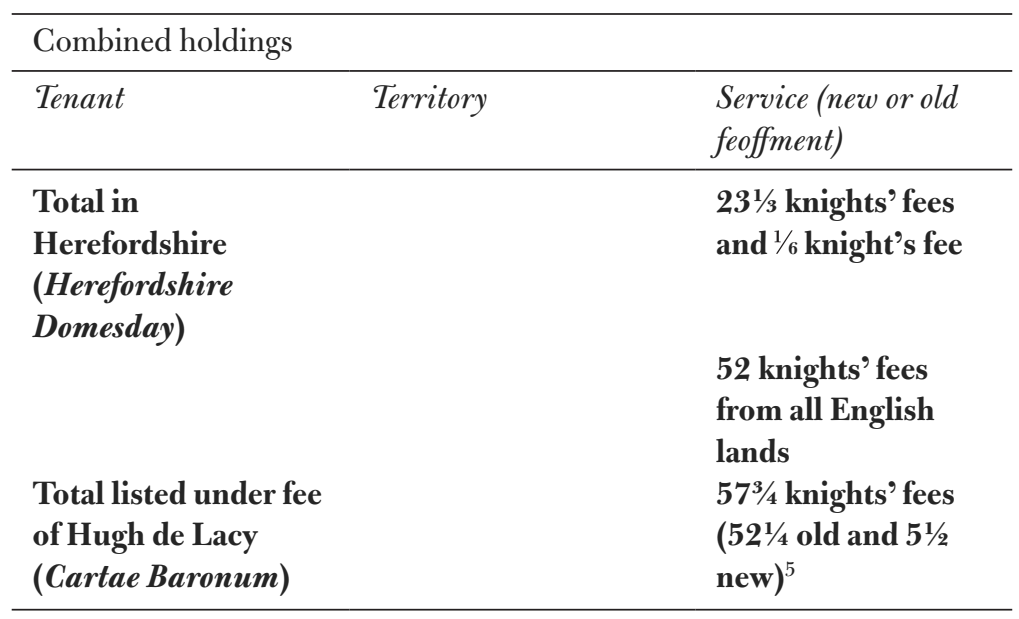

* Witnessed Walter de Lacy charter.

+ Family witnessed Walter de Lacy charter. 
Table 2: Tenants of Walter de Lacy in England

From list of fees, $1242-3^{6}$

\begin{tabular}{|c|c|c|c|}
\hline \multicolumn{4}{|l|}{ Herefordshire } \\
\hline Tenant & Territory & $\begin{array}{l}\text { Service (new or } \\
\text { old feoffment) }\end{array}$ & Sub-tenant(s) \\
\hline \multirow[t]{6}{*}{$\begin{array}{c}\text { *Baskerville, } \\
\text { Walter de }\end{array}$} & $\begin{array}{l}\text { Bodenham Rogeri } \\
\text { (1/2 hide) }\end{array}$ & 1/10 knight's fee & $\begin{array}{l}\text { +Bodenham, } \\
\text { Roger of }\end{array}$ \\
\hline & Brobury & $\begin{array}{l}1 / 5 \text { knight's fee } \\
\text { (old) }\end{array}$ & $\begin{array}{l}\text { Brobury, } \\
\text { Walter of }\end{array}$ \\
\hline & Letton (3 hides) & $\begin{array}{l}1 \text { knight's fee } \\
\text { (old) }\end{array}$ & $\begin{array}{l}\text { Horkesley } \\
\text { (Hurteslege), } \\
\text { Richard of }\end{array}$ \\
\hline & $\begin{array}{l}\text { Stretton Sugwas } \\
\text { ( } 1 \text { hide) }\end{array}$ & 1/5 knight's fee & \\
\hline & Yazor (2 hides) & $\begin{array}{l}2 / 3 \text { knight's fee } \\
\text { (old) }\end{array}$ & \\
\hline & Wacton (2 hides) & $\begin{array}{l}1 / 2 \text { knight's fee } \\
\text { (old) }\end{array}$ & $\begin{array}{l}\text { Cardiff, } \\
\text { William of }\end{array}$ \\
\hline $\begin{array}{l}\text { Beaufour, } \\
\text { Richard de }\end{array}$ & $\begin{array}{l}\text { Stoke Lacy (21/2 } \\
\text { hides) }\end{array}$ & $\begin{array}{l}\text { No service } \\
\text { (old) }\end{array}$ & \\
\hline $\begin{array}{l}\text { Bickerton, John } \\
\text { of }\end{array}$ & $\begin{array}{l}\text { Bickerton } \\
\text { ( } 1 \text { hide })\end{array}$ & $\begin{array}{l}1 / 2 \text { knight's fee } \\
\text { (old) }\end{array}$ & \\
\hline $\begin{array}{c}\text { +Bodenham, } \\
\text { Walter of }\end{array}$ & Byford & $\begin{array}{l}1 \text { knight's fee } \\
\text { (old) (with } \\
\text { John de Kenell' } \\
\text { and Matilda de } \\
\text { Tregoz) }\end{array}$ & \\
\hline $\begin{array}{l}\text { Caple, William } \\
\text { of }\end{array}$ & Wormsley (1 hide) & $\begin{array}{l}1 / 5 \text { knight's fee } \\
\text { (old) }\end{array}$ & \\
\hline $\begin{array}{c}\text { *+Criquetot, } \\
\text { Michael de } \\
\text { (heirs of) }\end{array}$ & $\begin{array}{l}\text { Webton, Cobhall, } \\
\text { La Mare }\end{array}$ & $\begin{array}{l}1 \text { knight's fee } \\
\text { (old) (Not in } \\
\text { final list of Lacy } \\
\text { fees) }\end{array}$ & \\
\hline $\begin{array}{l}\text { Englishman, } \\
\text { Roger the }\end{array}$ & Wolferlow & 1 knight's fee & \\
\hline $\begin{array}{l}+ \text { Escotot } \\
\qquad \begin{array}{l}\text { Scotot }), \\
\text { Walter de } \\
\text { (heirs of) }\end{array}\end{array}$ & Howton & 1 knight's fee & \\
\hline $\begin{array}{c}\text { +d'Évreux, } \\
\text { Cecilia }\end{array}$ & Putley & 1/5 knight's fee & \\
\hline
\end{tabular}


Table 2: (continued)

\begin{tabular}{|c|c|c|c|}
\hline \multicolumn{4}{|l|}{ Herefordshire } \\
\hline Tenant & Territory & $\begin{array}{l}\text { Service (new or } \\
\text { old feoffment) }\end{array}$ & Sub-tenant(s) \\
\hline & $\begin{array}{l}\text { Street (Lestret in } \\
\text { Kingsland) }\end{array}$ & $\begin{array}{l}1 / 3 \text { knight's fee } \\
\text { (old) }\end{array}$ & $\begin{array}{l}\text { Street, } \\
\text { Thomas of } \\
(\text { de la Strete })\end{array}$ \\
\hline $\begin{array}{c}+ \text { d'Évreux, } \\
\text { Isabella } \\
\text { (held in } \\
\text { dower) }\end{array}$ & $\begin{array}{l}\text { Halmond's Frome } \\
\text { ( } 4 \text { hides) }\end{array}$ & $\begin{array}{l}\text { 1/2 knight's fee } \\
\text { (old) (1 knight's } \\
\text { fee, relaxed by } \\
\text { half a fee by writ } \\
\text { of Walter de } \\
\text { Lacy to Stephen } \\
\text { d'Évreux) }\end{array}$ & \\
\hline $\begin{array}{l}* \text { +d'Évreux, } \\
\text { Nicholas } \\
\text { (Colinus in } \\
\text { original } \\
\text { hundred } \\
\text { return) }\end{array}$ & $\begin{array}{l}\text { Chanston (near } \\
\text { Vowchurch) }\end{array}$ & $\begin{array}{l}1 \text { knight's fee } \\
\text { (old) }\end{array}$ & \\
\hline $\begin{array}{c}+ \text { d'Évreux, } \\
\text { William }\end{array}$ & Holme Lacy & $\begin{array}{l}\text { Moiety of } 1 / 2 \\
\text { knight's fee (old) } \\
\text { (other moiety } \\
\text { held by heirs of } \\
\text { Walter de Lacy) } \\
\text { (Not in final list } \\
\text { of Lacy fees) }\end{array}$ & \\
\hline & Lyonshall, Upcott & $\begin{array}{l}1 \text { knight's fee } \\
\text { (old) }\end{array}$ & \\
\hline & $\begin{array}{l}\text { Stoke Lacy }(61 / 2 \\
\text { hides })\end{array}$ & $\begin{array}{l}1 / 4 \text { knight's fee } \\
\text { (old) }\end{array}$ & \\
\hline $\begin{array}{l}\text { Eynsford, } \\
\text { Walter de }\end{array}$ & $\begin{array}{l}\text { Pyon (Moiety of } 5 \\
\text { hides) }\end{array}$ & $\begin{array}{l}1 \text { knight's fee } \\
\text { (with Hugh de } \\
\text { Verlai) }\end{array}$ & \\
\hline $\begin{array}{l}*+\text { Feipo, } \\
\quad \text { Richard de }\end{array}$ & $\begin{array}{l}\text { Lyde Mucegros (1/2 } \\
\text { hide) }\end{array}$ & $\begin{array}{l}1 / 10 \text { knight's fee } \\
\text { (old) }\end{array}$ & \\
\hline $\begin{array}{l}+ \text { Feipo, } \\
\text { Thomas de }\end{array}$ & $\begin{array}{l}\text { Munsley } \\
\text { (1 moiety of } 3 \\
\text { hides) }\end{array}$ & $\begin{array}{l}1 / 2 \text { knight's fee } \\
\text { (old) (with } \\
\text { Henry of } \\
\text { Woodhall) }\end{array}$ & \\
\hline
\end{tabular}


Table 2: (continued)

\begin{tabular}{|c|c|c|c|}
\hline \multicolumn{4}{|l|}{ Herefordshire } \\
\hline Tenant & Territory & $\begin{array}{l}\text { Service (new or } \\
\text { old feoffment) }\end{array}$ & Sub-tenant(s) \\
\hline $\begin{array}{l}*+\text { Fourches, } \\
\text { William de }\end{array}$ & $\begin{array}{l}\text { Bodenham } \\
\text { (1 hide) }\end{array}$ & $\begin{array}{l}1 / 4 \text { knight's fee } \\
\text { (old) }\end{array}$ & $\begin{array}{l}+ \text { Fourches, } \\
\text { Isabella de } \\
\text { (held in } \\
\text { dower of } \\
\text { William) }\end{array}$ \\
\hline $\begin{array}{l}\text { Gamaches, } \\
\text { Godfrey de }\end{array}$ & Mansel Gamage & $\begin{array}{l}1 \text { knight's fee } \\
\text { (old) }\end{array}$ & \\
\hline \multirow[t]{2}{*}{$\begin{array}{l}\text { Gloucester, St } \\
\text { Peter's Abbey }\end{array}$} & $\begin{array}{l}\text { Monkhide } \\
\text { (1 hide) }\end{array}$ & Alms & \\
\hline & Lyde Saucy & $\begin{array}{l}1 / 2 \text { knight's fee } \\
\text { (Bought from } \\
\text { Ralph de } \\
\text { Saussey) }\end{array}$ & \\
\hline $\begin{array}{l}\text { Grendon, } \\
\text { John of }\end{array}$ & $\begin{array}{l}\text { Grendon } \\
\text { (1 hide) }\end{array}$ & $1 / 4$ knight's fee & \\
\hline \multirow[t]{5}{*}{$\begin{array}{l}\text { *Hampton, } \\
\text { Richard of }\end{array}$} & Bacton & $\begin{array}{l}1 \text { knight's fee } \\
\text { (old) }\end{array}$ & \\
\hline & $\begin{array}{l}\text { Hampton } \\
{[\text { Court }](2 \text { hides })}\end{array}$ & $\begin{array}{l}3 / 4 \text { knight's fee } \\
\text { (old) }\end{array}$ & \\
\hline & $\begin{array}{l}\text { Lawton (in } \\
\text { Kingswood) } \\
\text { (1 hide) }\end{array}$ & $\begin{array}{l}1 / 5 \text { knight's fee } \\
\text { (old) }\end{array}$ & $\begin{array}{l}\text { +d'Évreux, } \\
\text { William }\end{array}$ \\
\hline & Ledicot (1 hide) & $\begin{array}{l}1 / 5 \text { knight's fee } \\
\text { (old) }\end{array}$ & $\begin{array}{l}\text { Offerton } \\
\text { (Aufertun'), } \\
\text { Robert of } \\
\text { and Adam } \\
\text { fitz Maelis }\end{array}$ \\
\hline & $\begin{array}{l}\text { Mintridge } \\
\text { (1 hide) }\end{array}$ & $\begin{array}{l}1 / 4 \text { knight's fee (old) } \\
\text { (original hundred } \\
\text { return lists service } \\
\text { as } 1 / 5 \text { knight's fee) }\end{array}$ & $\begin{array}{l}\text { Mintridge, } \\
\text { Walter }\end{array}$ \\
\hline $\begin{array}{l}\text { Hereford, } \\
\text { Canons of } \\
\text { Hereford } \\
\text { Cathedral }\end{array}$ & Holme Lacy & $\begin{array}{l}1 \frac{1}{2} \text { knight's fee } \\
\text { (new) (Lacy held } \\
\text { of the bishop of } \\
\text { Hereford, so not } \\
\text { in final list of fees) }\end{array}$ & \\
\hline
\end{tabular}


Table 2: (continued)

\begin{tabular}{|c|c|c|c|}
\hline \multicolumn{4}{|l|}{ Herefordshire } \\
\hline Tenant & Territory & $\begin{array}{l}\text { Service (new or } \\
\text { old feoffment) }\end{array}$ & Sub-tenant(s) \\
\hline $\begin{array}{l}\text { Hereford, St } \\
\text { Guthlac's } \\
\text { Priory }\end{array}$ & $\begin{array}{l}\text { Leadon (near } \\
\text { Bishop's Frome) } \\
\text { (1/2 hide) }\end{array}$ & $\begin{array}{l}\text { No service } \\
\text { (old) }\end{array}$ & \\
\hline $\begin{array}{l}\text { Hurstley, } \\
\text { Richard of }\end{array}$ & $\begin{array}{l}\text { Whitwick } \\
\text { (1 moiety) }\end{array}$ & $\begin{array}{l}\text { 1/5 knight's fee } \\
\text { (old) }\end{array}$ & $\begin{array}{l}\text { Hide, } \\
\text { Richard of }\end{array}$ \\
\hline Kaerdin & $\begin{array}{l}\text { Villa Ricardi } i^{7}(1 \\
\text { moiety of } 1 \text { hide })\end{array}$ & $\begin{array}{l}1 / 5 \text { knight's fee } \\
\text { (old) }\end{array}$ & $\begin{array}{l}\text { Hide, } \\
\text { Richard of }\end{array}$ \\
\hline $\begin{array}{l}+ \text { Kenell',John } \\
\quad \text { de }\end{array}$ & Byford & $\begin{array}{l}1 \text { knight's fee } \\
\text { (old) (with } \\
\text { Matilda de } \\
\text { Tregoz and } \\
\text { Walter of } \\
\text { Bodenham) }\end{array}$ & \\
\hline $\begin{array}{l}\text { +Lacy, Gilbert } \\
\quad \text { de }\end{array}$ & $\begin{array}{l}\text { Castle Frome } \\
\text { ( } 4 \text { hides) }\end{array}$ & $\begin{array}{l}1 \text { knight's fee } \\
\text { (old) }\end{array}$ & \\
\hline $\begin{array}{l}*+\text { Lacy, Margery } \\
\text { de }\end{array}$ & $\begin{array}{l}\text { Weobley } \\
(5 \text { hides })\end{array}$ & 1 knight's fee & \\
\hline $\begin{array}{l}\text { Landa, Henry } \\
\text { (heirs of) }\end{array}$ & Wyle (2 hides) & $\begin{array}{l}\text { 1/4 knight's fee } \\
\text { (Original } \\
\text { hundred return } \\
\text { records that } \\
\text { this service is } \\
\text { owed to the } \\
\text { honor of Castle } \\
\text { Richard) }\end{array}$ & \\
\hline Llanthony Priory & $\begin{array}{l}\text { Yarsop ( } 1 \text { hide) } \\
\text { Canon Frome } \\
\text { ( } 3 \text { hides })\end{array}$ & $\begin{array}{l}\text { Socage } \\
\text { No service } \\
(\text { elemosina })\end{array}$ & \\
\hline $\begin{array}{l}\text { Malory } \\
\text { (Malhore), } \\
\text { Robert }\end{array}$ & $\begin{array}{l}\text { Lyde Saucey } \\
\text { (1 hide) }\end{array}$ & Socage & \\
\hline $\begin{array}{l}\text { *Monmouth, } \\
\text { John of } \\
\text { +Muscegros, } \\
\text { Amabilia and } \\
\text { Juliana de }\end{array}$ & $\begin{array}{l}\text { Whitwick } \\
\text { ( } 2 \text { hides) } \\
\text { Lyde Mucegros } \\
\text { (1/2 hide) }\end{array}$ & $\begin{array}{l}\text { 1/2 knight's fee } \\
\text { (old) } \\
1 / 10 \text { knight's fee } \\
\text { (old) }\end{array}$ & $\begin{array}{l}\text { Hurstley, } \\
\text { Richard of }\end{array}$ \\
\hline
\end{tabular}


Table 2: (continued)

\begin{tabular}{|c|c|c|c|}
\hline \multicolumn{4}{|l|}{ Herefordshire } \\
\hline Tenant & Territory & $\begin{array}{l}\text { Service (new or } \\
\text { old feoffment) }\end{array}$ & Sub-tenant(s) \\
\hline \multirow[t]{5}{*}{$\begin{array}{c}*+\text { Pichard } \\
\text { Roger }\end{array}$} & Almeley (4 hides) & $\begin{array}{l}1 \text { knight's fee } \\
\text { (old) }\end{array}$ & \\
\hline & $\begin{array}{l}\text { Ocle Pychard } \\
\text { ( } 4 \text { hides) }\end{array}$ & $\begin{array}{l}3 / 4 \text { knight's fee } \\
\text { (old) (original } \\
\text { hundred return } \\
\text { lists } 2 / 3 \text { knight's } \\
\text { fee) }\end{array}$ & \\
\hline & Stanford (1 hide) & 1/6 knight's fee & $\begin{array}{l}\text { Stanford, } \\
\text { Walter of }\end{array}$ \\
\hline & $\begin{array}{l}\text { Staunton-on-Wye } \\
\text { (4 hides) }\end{array}$ & 1 knight's fee & $\begin{array}{l}\text { *+Pichard, } \\
\text { Miles }\end{array}$ \\
\hline & $\begin{array}{l}\text { Whitechurch } \\
\text { Maun ( } 2 \text { hides) }\end{array}$ & $\begin{array}{l}1 / 4 \text { knight's fee } \\
\text { (old) }\end{array}$ & $\begin{array}{l}\text { +d'Évreux, } \\
\text { Isabella }\end{array}$ \\
\hline $\begin{array}{c}*+\text { Pichard } \\
\text { Miles }\end{array}$ & Marston (2 hides) & $\begin{array}{l}1 / 2 \text { knight's fee } \\
\text { (old) }\end{array}$ & $\begin{array}{l}\text { +Pichard, } \\
\text { William }\end{array}$ \\
\hline $\begin{array}{l}\text { Ragun, John } \\
\text { son of Hugh }\end{array}$ & $\begin{array}{l}\text { Chanston (near } \\
\text { Vowchurch) }\end{array}$ & $\begin{array}{l}1 / 6 \text { knight's fee } \\
\text { (old) }\end{array}$ & \\
\hline \multirow[t]{3}{*}{$\begin{array}{l}\text { Sarnesfield, } \\
\text { Philip de }\end{array}$} & $\begin{array}{l}\text { Marston ( } 1 \text { moiety } \\
\text { of } 1 \text { hide) }\end{array}$ & $\begin{array}{l}\text { 1/4 knight's fee } \\
\text { (old) (with } \\
\text { heirs of Walter } \\
\text { of Traneley) }\end{array}$ & $\begin{array}{l}\text { Grendon, } \\
\text { John of } \\
\text { Subinfeu- } \\
\text { dated to: } \\
\text { +Bradley, } \\
\text { Andreas of }\end{array}$ \\
\hline & Sarnesfield & $\begin{array}{l}1 / 2 \text { knight's fee } \\
\text { (old) }\end{array}$ & \\
\hline & Swanston (1 hide) & $\begin{array}{l}1 / 3 \text { knight's fee } \\
\text { (old) }\end{array}$ & \\
\hline $\begin{array}{c}*+\text { Saussey, } \\
\text { Ralph de }\end{array}$ & $\begin{array}{l}\text { Wormeton (in } \\
\text { Kilpeck) }\end{array}$ & $\begin{array}{l}1 / 2 \text { knight's fee } \\
\text { (old) }\end{array}$ & \\
\hline $\begin{array}{l}\text { Tosny, } \\
\text { Petronilla de } \\
\text { (née de Lacy) }\end{array}$ & Yarkhill (4 hides) & $\begin{array}{l}1 \text { knight's fee } \\
\text { (old) }\end{array}$ & \\
\hline $\begin{array}{l}\text { Traneley, } \\
\text { Walter of } \\
\text { (heirs of) }\end{array}$ & $\begin{array}{l}\text { Marston ( } 1 \text { moiety } \\
\text { of } 1 \text { hide) }\end{array}$ & $\begin{array}{l}\text { 1/4 knight's fee } \\
\text { (old) (with } \\
\text { Sarnesfield, } \\
\text { Philip de) }\end{array}$ & $\begin{array}{l}\text { Wintercott, } \\
\text { John of }\end{array}$ \\
\hline
\end{tabular}


Table 2: (continued)

\begin{tabular}{|c|c|c|c|}
\hline \multicolumn{4}{|l|}{ Herefordshire } \\
\hline Tenant & Territory & $\begin{array}{l}\text { Service (new or } \\
\text { old feoffment) }\end{array}$ & Sub-tenant(s) \\
\hline $\begin{array}{l}\text { +Tregoz, } \\
\text { Matilda de }\end{array}$ & Byford & $\begin{array}{l}1 \text { knight's fee } \\
\text { (old) (with } \\
\text { John de Kenell' } \\
\text { and Walter of } \\
\text { Bodenham) }\end{array}$ & \\
\hline \multirow[t]{3}{*}{ +Tyrell, Roger } & $\begin{array}{l}\text { Evesbatch } \\
\text { (1 hide) } \\
\text { Little Marcle }\end{array}$ & $\begin{array}{l}\text { 1/5 knight's fee } \\
\text { (old) } \\
1 \text { knight's fee } \\
\text { (old) }\end{array}$ & $\begin{array}{l}\text { Evesbatch, } \\
\text { Stephen of }\end{array}$ \\
\hline & $\begin{array}{l}\text { Maund } \\
\text { (Magheme) } \\
\text { (1 } 12 \text { hide) }\end{array}$ & $\begin{array}{l}\text { 1/10 knight's fee } \\
\text { (old) }\end{array}$ & $\begin{array}{l}\text { Hammond, } \\
\text { Walter }\end{array}$ \\
\hline & $\begin{array}{l}\text { Wormeton (in } \\
\text { Kilpeck) }\end{array}$ & $\begin{array}{l}1 / 2 \text { knight's fee } \\
\text { (old) }\end{array}$ & \\
\hline Verlai, Hugh de & $\begin{array}{l}\text { Pyon (Moiety of } 5 \\
\text { hides) }\end{array}$ & $\begin{array}{l}1 \text { knight's fee } \\
\text { (with Walter of } \\
\text { Eynsford) }\end{array}$ & \\
\hline $\begin{array}{l}\text { Weston, Robert } \\
\text { of }\end{array}$ & $\begin{array}{l}\text { Weston Beggard } \\
\text { (1 hide) }\end{array}$ & $\begin{array}{l}1 / 5 \text { knight's fee } \\
\text { (old) }\end{array}$ & \\
\hline $\begin{array}{l}\text { Woodhall, } \\
\text { Henry of }\end{array}$ & $\begin{array}{l}\text { Munsley (Moiety } \\
\text { of } 3 \text { hides) }\end{array}$ & $\begin{array}{l}\text { 1/2 knight's fee } \\
\text { (old) (with } \\
\text { Thomas de } \\
\text { Feipo) }\end{array}$ & \\
\hline $\begin{array}{l}\text { *Wormsley, } \\
\text { Walter of }\end{array}$ & $\begin{array}{l}\text { Staunton-on-Wye } \\
\text { ( } 2 \text { hides) }\end{array}$ & $\begin{array}{l}1 / 2 \text { knight's fee } \\
\text { (old) }\end{array}$ & \\
\hline $\begin{array}{l}\text { *Elias the clerk, } \\
\text { Richard the } \\
\text { cook, Richard } \\
\text { Caperun and } \\
\text { William le Bel }\end{array}$ & $\begin{array}{l}\text { Whyle (Moiety of } \\
1 \text { hide) }\end{array}$ & $\begin{array}{l}1 / 4 \text { knight's fee } \\
\text { (old) }\end{array}$ & \\
\hline $\begin{array}{l}\text { Total listed } \\
\text { under fee of } \\
\text { Walter de } \\
\text { Lacy }\end{array}$ & & $\begin{array}{l}271 / 2 \text { knights } \\
\text { fees and } 1 / 5 \\
\text { knight's fee } \\
\text { and } 1 / 24 \text { knight's } \\
\text { fee }\end{array}$ & \\
\hline
\end{tabular}


Table 2: (continued)

\begin{tabular}{|c|c|c|c|}
\hline \multicolumn{4}{|l|}{ Shropshire } \\
\hline Tenant & Territory & $\begin{array}{l}\text { Service (new or } \\
\text { old feoffment) }\end{array}$ & Sub-tenant(s) \\
\hline \multirow{2}{*}{$\begin{array}{l}\text { Bitterley, } \\
\text { Stephen of }\end{array}$} & Bitterley & 1 knight's fee & \\
\hline & Rushbury & $\begin{array}{l}1 \text { knight's fee } \\
\text { (with Petronilla } \\
\text { de Kenley) }\end{array}$ & \\
\hline $\begin{array}{l}\text { Clinton, Philip } \\
\text { of }\end{array}$ & Henley & $1 / 2$ knight's fee & \\
\hline $\begin{array}{c}\text { *+Criquetot, } \\
\text { Michael de } \\
\text { (heirs of) }\end{array}$ & Upper Hayton & $1 / 2$ knight's fee & \\
\hline $\begin{array}{c}\text { +d'Évreux, } \\
\text { William }\end{array}$ & Lower Hayton & $1 / 2$ knight's fee & \\
\hline $\begin{array}{c}*_{+}+\text {Fourches, } \\
\text { William de }\end{array}$ & Corfton & 1 knight's fee & \\
\hline $\begin{array}{l}\text { Hopton, } \\
\quad \text { Nicholas of }\end{array}$ & Hopton Waffers & 1 knight's fee & \\
\hline $\begin{array}{l}\text { Kenley, } \\
\text { Petronilla de } \\
\text { (dau. of } \\
\text { Roger fitz } \\
\text { Odo) }\end{array}$ & Rushbury & $\begin{array}{l}1 \text { knight's fee } \\
\text { (with Stephen } \\
\text { de Bitterley) }\end{array}$ & \\
\hline $\begin{array}{l}\text { Mauduit, } \\
\text { Thomas }\end{array}$ & $\begin{array}{l}\text { Great Sutton, } \\
\text { Witchcot }\end{array}$ & 1 knight's fee & \\
\hline +Piron, John & Pole & $1 / 4$ knight's fee & \\
\hline $\begin{array}{c}*+\text { Poer, Roger } \\
\text { le and his } \\
\text { associates }\end{array}$ & Aldon & $1 / 2$ knight's fee & \\
\hline Sai, Walter de & $\begin{array}{l}\text { Stokesay, Stoke- } \\
\text { upon-Tern, } \\
\text { Wheathill }\end{array}$ & 3 knights' fees & \\
\hline $\begin{array}{l}\text { Wootton, } \\
\text { Robert of }\end{array}$ & Wootton & $1 / 2$ knight's fee & \\
\hline Total & & $\begin{array}{l}10^{3} / 4 \text { knights' } \\
\text { fees }\end{array}$ & \\
\hline
\end{tabular}


Table 2: (continued)

\begin{tabular}{|c|c|c|c|}
\hline \multicolumn{4}{|l|}{ Oxfordshire } \\
\hline Tenant & Territory & $\begin{array}{l}\text { Service (new or } \\
\text { old enfeoffment) }\end{array}$ & Sub-tenant(s) \\
\hline $\begin{array}{l}\text { Pirneho, Robert } \\
\quad \text { de }\end{array}$ & $\begin{array}{l}\text { Barford (Bereford } \\
\text { Olof) }\end{array}$ & $1 / 2$ knight's fee & \\
\hline \multirow[t]{2}{*}{$\begin{array}{c}\text { *+Saussey, } \\
\text { Ralph de }\end{array}$} & Begbroke & 1 knight's fee & $\begin{array}{l}\text { Leuns, } \\
\text { Matilda de } \\
\text { and Studley, } \\
\text { Prioress of }\end{array}$ \\
\hline & $\begin{array}{l}\text { Sandford, } \\
\text { Cornwell }\end{array}$ & $1 / 2$ knight's fee & \\
\hline $\begin{array}{l}\text { +Saussey, } \\
\quad \text { Leticia de }\end{array}$ & $\begin{array}{l}\text { Nethercote } \\
\text { (Nethercudinton) }\end{array}$ & $1 / 2$ knight's fee & \\
\hline Total & & $\begin{array}{l}2^{1 / 2} \text { knights' } \\
\text { fees }\end{array}$ & \\
\hline
\end{tabular}

Gloucestershire, Bailiwick of Cirencester (from 1242 Scutage for Gascony)

\begin{tabular}{|c|c|c|c|}
\hline Tenant & Territory & $\begin{array}{l}\text { Service (new or } \\
\text { old enfeoffment) }\end{array}$ & Sub-tenant(s) \\
\hline $\begin{array}{l}\text { +d'Évreux, } \\
\text { Cecilia }\end{array}$ & Eastleach Turville & $\begin{array}{l}2 \text { knights' fees } \\
\text { (with Galliana } \\
\text { de Tourville) }\end{array}$ & \\
\hline $\begin{array}{l}\text { Coates, Ranulf } \\
\text { of }\end{array}$ & Coates & 1 knight's fee & \\
\hline $\begin{array}{l}\text { *Hampton, } \\
\text { Richard of }\end{array}$ & Stratton & 1 knight's fee & \\
\hline $\begin{array}{l}\text { Langley, } \\
\text { Geoffrey de }\end{array}$ & Siddington & $1 / 2$ knight's fee & \\
\hline $\begin{array}{l}\text { Tourville, } \\
\text { Galliana de }\end{array}$ & Eastleach Turville & $\begin{array}{l}2 \text { knights' fees } \\
\text { (with Cecilia } \\
\text { d'Évreux) }\end{array}$ & \\
\hline Total & & $\begin{array}{l}4 \frac{1}{2} \text { knights' } \\
\text { fees }\end{array}$ & \\
\hline
\end{tabular}

* Witnessed Walter de Lacy charter.

+ Family witnessed Walter de Lacy charter. 
Table 3: The subinfeudation of Meath

From The Song of Dermot and the Earl ${ }^{9}$

\begin{tabular}{|c|c|c|}
\hline Tenant & Territory & Service (if known) \\
\hline $\begin{array}{l}\text { Angulo, Jocelin } \\
\text { de }\end{array}$ & $\begin{array}{l}\text { Ardbraccan (bar. Lower Navan, Co. } \\
\text { Meath) Navan (Co. Meath) }\end{array}$ & \\
\hline $\begin{array}{l}\text { Angulo, Gilbert } \\
\text { de }\end{array}$ & Morgallion (Co. Meath) & \\
\hline $\begin{array}{l}\text { *+Capella, } \\
\text { Richard de }\end{array}$ & Unspecified 'rich fief' & \\
\hline $\begin{array}{l}\text { Costentin, } \\
\text { Geoffrey de }\end{array}$ & Kilbixy (Co. Westmeath) & \\
\hline Graville, & Emlagh (bar. Lower Kells, Co. & \\
\hline Thomas de ${ }^{10}$ & $\begin{array}{l}\text { Meath) Laragh and Shanonagh } \\
\text { (bar. Moygoish, Co. Westmeath) }\end{array}$ & \\
\hline Dullard, Adam & $\begin{array}{l}\text { Rathconarty (incl. Dollardstown } \\
\text { and Painestown, bar. Duleek, Co. } \\
\text { Meath) }\end{array}$ & \\
\hline *+Feipo, Adam & Skreen (Co. Meath) Santry and & 20 knights' fees; 1 \\
\hline de & Clontarf (Co. Dublin) & knight's fee \\
\hline $\begin{array}{l}\text { +Fleming, } \\
\text { Richard le }\end{array}$ & Slane (Co. Meath) & 20 knights' fees \\
\hline $\begin{array}{l}*+\text { Hose, Hugh } \\
\quad \text { de }\end{array}$ & Deece (Co. Meath) & \\
\hline $\begin{array}{l}*+\text { Lacy, Robert } \\
\quad \text { de }\end{array}$ & Rathwire (Co. Meath) & \\
\hline $\begin{array}{l}\text { *Meiler fitz } \\
\text { Henry }\end{array}$ & Ardnurcher (Co. Westmeath) & \\
\hline $\begin{array}{l}\text { *Meset, William } \\
\text { de }\end{array}$ & Lune (Co. Meath) & \\
\hline $\begin{array}{l}\text { *Nugent, } \\
\text { Gilbert de }\end{array}$ & Delvin (Co. Westmeath) & 5 knights' fees \\
\hline *+Petit, William & Chastelbrec (Castle Bret?) & 1 knight for every \\
\hline vle & $\begin{array}{l}\text { Magheradernon (Co. Westmeath) } \\
\text { Rathkenny (Co. Meath) Leulkeil } \\
\text { and Kleonkelli (bar. Shrule, Co. } \\
\text { Longford) }\end{array}$ & $\begin{array}{l}30 \text { carucates, } \\
\text { performed at Lacy } \\
\text { castle of Killare } \\
\text { (Co. Westmeath) }\end{array}$ \\
\hline $\begin{array}{l}\text { *+Tuit, Richard } \\
\text { de }\end{array}$ & Granard (Co. Longford) & \\
\hline *+Tyrell, Hugh & Castleknock (Co. Dublin) & \\
\hline
\end{tabular}

* Witnessed Hugh or Walter de Lacy charter.

+ Family witnessed Hugh or Walter de Lacy charter. 
Table 4: Assorted Lacy knights and officials

\begin{tabular}{|c|c|c|}
\hline \multicolumn{3}{|l|}{ Hugh de Lacy } \\
\hline Name & Position & Dates \\
\hline Richard le Bret ${ }^{11}$ & Retainer & 1166 \\
\hline Ralph de Riqueot & Retainer & 1166 \\
\hline Adam de Feipo & Retainer & 1166 \\
\hline Anketil de Escotot & Retainer & 1166 \\
\hline William de Escotot & Retainer & 1166 \\
\hline Son of Gerard de Macgne & Retainer & 1166 \\
\hline Roger de Escorchebeuf & Retainer & 1166 \\
\hline Bartholomaeus & Retainer & 1166 \\
\hline Hugh Tyrell ${ }^{12}$ & $\begin{array}{l}\text { Castellan of Trim and } \\
\text { Duleek }\end{array}$ & $1172-1173$ \\
\hline Richard of Stottesdon ${ }^{13}$ & Chamberlain & $c .1186$ \\
\hline \multicolumn{3}{|l|}{ Walter de Lacy } \\
\hline Name & Position & Dates \\
\hline William de Briouze ${ }^{14}$ & $\begin{array}{l}\text { Seneschal in England } \\
\text { and Normandy }\end{array}$ & $1202-c .1207$ \\
\hline Milo Pichard ${ }^{15}$ & Bailiff & 1208 \\
\hline William le Petit ${ }^{16}$ & Constable of Meath & $c .1202 \times 1210$ \\
\hline William 'Gorm' de Lacy ${ }^{17}$ & Seneschal of Meath & 1215 -post 1217 \\
\hline Thomas d'Anisy $^{18}$ & Shrieval Deputy & $\begin{array}{l}1218-1219 \\
1222-1223\end{array}$ \\
\hline \multirow[t]{2}{*}{ Richard de Fay ${ }^{19}$} & Knight & $1219 \times 1220$ \\
\hline & Chancellor & $1202 \times 1231$ \\
\hline $\begin{array}{l}\text { Warin de Grentemesnil } \\
\qquad(\text { Grendon })^{20}\end{array}$ & Shrieval Deputy & $1220-1221$ \\
\hline Walter of Caldicot ${ }^{21}$ & Seneschal & Post 1216 \\
\hline Nicholas fitz Leo ${ }^{22}$ & Seneschal of Meath & $c .1219-c .1221$ \\
\hline Simon of Clifford ${ }^{23}$ & Seneschal & $c .1231$ \\
\hline Richard of Copeland ${ }^{24}$ & $\begin{array}{l}\text { Retained Knight } \\
\text { (milite mei) }\end{array}$ & c.9 April 1234 \\
\hline John of Copeland & $\begin{array}{l}\text { Retained Knight } \\
\text { (milite mei) }\end{array}$ & c.9 April 1234 \\
\hline Walter le Petit & $\begin{array}{l}\text { Retained Knight } \\
\text { (milite mei) }\end{array}$ & c.9 April 1234 \\
\hline Robert of Leyburn & $\begin{array}{l}\text { Retained Knight } \\
\text { (milite mei) }\end{array}$ & c.9 April 1234 \\
\hline
\end{tabular}


Table 4: (continued)

\begin{tabular}{lll}
\hline Walter de Lacy & & \\
\hline Name & Position & Dates \\
\hline Hugh, conon of Llanthony & Chaplain & $c .9$ April 1234 \\
Nicholas d'Ėvreux & Seneschal & $c .9$ April 1234 \\
Paul le Cornwalays & Chamberlain & $c .9$ April 1234 \\
Walter le Dispenser & Procurator of & $c .9$ April 1234 \\
& Donacarney & \\
John of Clifford & Servant & $c .9$ April 1234 \\
Philip de Wichetot & Servant & $c .9$ April 1234 \\
Brother Hugh of Stanton & Chancellor & 1 Aug 1234 \\
Philip de Colleville ${ }^{26}$ & Seneschal; Steward in & $1233 \times 123612$ \\
& England & June 1234 \\
William de Lucy ${ }^{27}$ & Steward in England; & 1 September \\
& constable of Ludlow & 1234 \\
& castle & \\
\hline
\end{tabular}


Table 5: Most frequent witnesses to Lacy acta

\begin{tabular}{|c|c|c|}
\hline \multicolumn{3}{|c|}{ Walter de Lacy - More than two attestations } \\
\hline Number & Witness & Other Lacy attestations \\
\hline 11 & William le Petit & $\begin{array}{l}1 \text { - Hugh de Lacy } \\
4 \text { - Hugh de Lacy the younger }\end{array}$ \\
\hline 10 & Simon of Clifford & 1 - Hugh de Lacy the younger \\
\hline 10 & Walter le Petit & \\
\hline 8 & Richard de Tuit & 6 - Hugh de Lacy the younger \\
\hline 7 & Nicholas d'Évreux & 1 - Katharine de Lacy \\
\hline 6 & William 'Gorm' de Lacy & 4 - Hugh de Lacy the younger \\
\hline 5 & $\begin{array}{l}\text { Richard de la Corner, } \\
\text { bp Meath }\end{array}$ & 2 - Hugh de Lacy the younger \\
\hline 5 & Walter of Caldicot & $\begin{array}{l}1 \text { - Margery de Lacy } \\
1 \text { - Gilbert de Lacy }\end{array}$ \\
\hline 5 & Simon of Tyleshope & \\
\hline 4 & Robert de Lacy & $\begin{array}{l}5 \text { - Hugh de Lacy the younger } \\
1 \text { - Katharine de Lacy }\end{array}$ \\
\hline 4 & $\begin{array}{l}\text { Hugh de Lacy the } \\
\text { younger }\end{array}$ & 1 - Gilbert de Lacy \\
\hline 4 & Richard de Feipo & 1 - Margery de Lacy \\
\hline 4 & Matthew de Tuit & 1 - Hugh de Lacy the younger \\
\hline 4 & Adam Fayel & \\
\hline 4 & Amaury de Lacy & \\
\hline 4 & Milo Pichard & \\
\hline 4 & Philip de Wichetot & \\
\hline 3 & Philip de Colleville & 1 - Margery de Lacy \\
\hline 3 & $\begin{array}{l}\text { Peter des Roches, bp. } \\
\text { Winchester }\end{array}$ & 1 - Gilbert de Lacy \\
\hline 3 & William de Fourches & 1 - Gilbert de Lacy \\
\hline 3 & $\begin{array}{l}\text { Ralph le Petit, adn. } \\
\text { Meath }\end{array}$ & 1 - Hugh de Lacy the younger \\
\hline 3 & William Talbot & \\
\hline 3 & Hugh Hose & \\
\hline 3 & John of Cranford & \\
\hline 3 & William Hachet & \\
\hline 3 & Robert of Fenhampton & \\
\hline 3 & William of Tregoyd & \\
\hline 3 & Ralph de Saussey & \\
\hline
\end{tabular}


Table 5: (continued)

\begin{tabular}{|c|c|c|}
\hline \multicolumn{3}{|c|}{ Hugh de Lacy - More than one attestation } \\
\hline Number & Witness & Other Lacy attestations \\
\hline 3 & William de Meset & \\
\hline 3 & Miles de Muscegros & \\
\hline 2 & Gilbert de Nugent & 1 - Walter de Lacy \\
\hline 2 & $\begin{array}{l}\text { William, son of William fitz } \\
\text { Alan }\end{array}$ & \\
\hline 2 & $\begin{array}{l}\text { Robert de Hautvilliers (Alto } \\
\text { Villari) }\end{array}$ & \\
\hline 2 & Robert de Baskerville & \\
\hline 2 & Robert de Bigarz & \\
\hline 2 & John d'Évreux & \\
\hline 2 & Nicholas de Dignon & \\
\hline 2 & Robert Marshal & \\
\hline 2 & William de Mineres & \\
\hline
\end{tabular}

\section{Notes}

1 Liber Niger, i, pp.153-5; RBE, i, pp.281-3; Herefordshire Domesday, circa 116o1170 , eds V. H. Galbraith and James Tait (London, 1950).

2 Hereford Domesday, p.48, simply lists 'Robert' as the tenant. This is most likely either Robert de Baskerville or Robert, ancestor of the Walter of Brobury who held Brobury of Walter de Baskerville in 1242-3 (see below).

3 Liber Niger, i, p.153. RBE, i, p.281, incorrectly lists the service as eight fees.

4 It is unclear from the marginal notation which manor is intended, Hereford Domesday, p. 51 .

5 The service of $3^{1 / 2}$ knights (old feoffment) was denied by tenants.

6 Liber Feodorum, ii, pp.797-840, 954 .

7 Gailbraith and Tait identify this with Monkhide, Herefordshire Domesday, p. 99 .

8 Newly held by Margery by command of the archbishop of York and the justiciar.

9 Song, lines 3132-77; Deeds, lines 3130-75. See also, Orpen, Normans, ii, chapter 15, 'The sub-infeudation of Meath'.

10 He witnessed a charter of Hugh the younger to Maurice fitz Gerald. The Red Book of the Earls of Kildare, ed. Gearóid Mac Niocaill (Dublin, 1964), no. 21.

11 For all of these 1166 retainers, Liber Niger, i, p. 155; RBE, i, p. 283.

12 Expugnatio Hibernica, p.140.

13 Register of the Hospital of S. Fohn the Baptist without the New Gate, Dublin, ed. E. St J. Brooks (Dublin, 1936), no. 191.

14 See Chapters 4 and 5 . 
15 Rot. Litt. Pat., p.91.

16 Irish Llanthony, pp. 74, 215-16.

17 See Chapters 5 and 6.

18 Pipe Roll 2 Henry III, p. 90; Pipe Roll 3 Henry III, p. 165; Pipe Roll 6 Hen III, p. 64; Pipe Roll 7 Hen III, p. 185 .

19 Morris, Chester, p.11; Cal. Doc. Ireland, 1171-1251, no. 934.

20 Pipe Roll 4 Henry III, p. 194; Pipe Roll 5 Henry III, p. 13.

21 TNA E315/55, fos $34 \mathrm{r}-\mathrm{v}$.

22 Rot. Litt. Claus., i, p.39ob; Cal. Pat. and Close, Ireland, p. 197.

23 Memoranda Roll 1230-1, p. 46.

24 For all of Walter's officials $c .9$ April 1234, Coucher Book of Furness, i, pp.18-20.

25 Vincent, 'Lucys', no 15, p. 43.

26 'Pilippo de Ecleuill' tunc senescallo meo', Somerset Record Office DD $\mathrm{SAS} \backslash \mathrm{H} / 438$, fo. 59r; Cal. Pat. Rolls, 1232-47, p. 56 .

27 Vincent, 'Lucys', no. 15, pp.41-3. 